\title{
Role of Radiotherapy in Carcinosarcoma Larynx - A Rare Case Scenario
}

\section{Dr Harish Kumar $\mathrm{K}^{\star, \dagger, 1}$, Dr Narendran ${ }^{2}$, Dr Selvaluxmy Ganesharaja ${ }^{3}$}

\author{
${ }^{1}$ Assistant professor, Department of Radiation oncology, Cancer Institute (WIA), India \\ ${ }^{2}$ Postgraduate - Final Year, Department of Radiation Ocology, Cancer Institute (WIA), India \\ ${ }^{3}$ Professor/HOD, Department of Radiation oncology, Cancer Institute (WIA), India \\ Q $_{\text {https://doi.org/10.15520/jcmro.v2i07.187 }}$
}

Accepted 26-07-2019; Received 23-07-2019; Publish Online 30-07-2019

\author{
Reviewed By: \\ Dr V Daniel \\ Department: \\ Reviewer/CMRO
}

\begin{abstract}
Carcinosarcoma is very rare entity comprises $<2 \%$ of all laryngeal carcinomas. It is a Biphasic tumour showing both carcinomatous (Epithelial component) and sarcomatous differentiation (Mesenchymal component). Here we present a case of 60 years old elderly male came with hoarseness of voice for 3 months diagnosed as Carcinosarcoma of Left vocal cord-Stage I ,treated with Definitive radiation therapy and voice preservation was achieved. Although most of cases are treated with laryngectomy, no definite treatment protocol has been made because of its rare presentation.
\end{abstract}

\section{INTRODUCTION:}

Carcinosarcoma of larynx is highly malignant variant of Squamous cell carcinoma(SCC), uncommon, compromises $<2 \%$ of all laryngeal carcinomas [1]. Also known as Pseudosarcoma, Sarcomatoid Squamous Cell Carcinoma, Polypoidal Squamous cell carcinoma. They are aggressive tumour with good prognosis, as they can be detected early stage due to obstructive symptoms like dyspnea, odynophagia and progressive hoarseness of voice. We here presenting our institute experience on treating Carcinosarcoma of Larynx-Left vocal cord cT1N0M0 with definite radiation therapy.

\section{CASE REPORT:}

An apparently healthy 60 years old elderly man evaluated for hoarseness of voice for 3 months duration which is insidious in onset, gradually progressive. Examination and investigation done at ENT clinic in local hospital showed nodular growth of $5 \mathrm{~mm}$ in left vocal cord biopsy done came as Malignant neoplasm with spindle and pleomorphic changes . Reported to our hospital for further management.

Patient had history of smoking for 20 years -1pack/day and chronic alcoholic for 20years with comorbid as Diabetes mellitus, Systemic hypertension, Dyslipidemia and

\footnotetext{
* Corresponding author.

† Email: hharish_mbbs@yahoo.co.in
}

Chronic obstructive pulmonary disease for 15 years duration on regular treatment. Had no family history of cancers, non-consanguineous marriage and taking mixed diet.

On clinical examination oral cavity and oropharynx revealed normal findings. Indirect laryngoscopy showed mild irregularity of left vocal cord with normal mobility. Right Vocal cord normal and mobile. RIMA glottis adequate. No significant lymph node present in neck.

Hemogram, Renal function test, Liver function test are normal. Viral markers done negative. Direct laryngoscopy Direct Pharyngoscopy(DLDP) done showed a small residual $3 \mathrm{~mm}$ nodular lesion in mid $3^{\text {rd }}$ of left vocal cord with irregularity in Posterior $1 / 3^{\text {rd }}$ of left vocal cord with normal mobility. Right vocal cord was normal and mobile. Left vocal cord Lesion removed in toto and sent for biopsy. Rest of structures were normal.

Computed tomography of neck showed irregularity of left vocal cord. No subglottic or paraglottic extension made out. No significant lymph nodes in neck Figure 1. Metastatic work up Chest X ray and Computed tomography of chest showed no lung parenchymal lesion.

Histopathology done specimen done shows squamous epithelium with an underlying neoplasm composed of single scattered malignant cells infiltrating the stroma. The cells are polygonal to spindle shaped with markedly pleomorphic nuclei with prominent nucleoi. Stroma shows myxoid degeneration focally suggestive of poorly differentiated Malignant tumour. Advised Immunohistochemistry(IHC) for Keratin, Vimentin, CK5/6, P40, SMA, Desmin, Caldesmon, CD 68, S100. IHC done showed positive for Keratin, Vimentin, 
CK5/6, P40, SMA and Negative for remaining IHC's .Final histopathology was suggestive of Poorly differentiated sarcoma, high grade with focal dysplasia of lining squamous epithelium(5). Possibility of carcinosarcoma. Outside paraffin block reviewed was also suggestive of carcinosarcoma.

After evaluation, diagnosed as a case of carcinosarcoma of Left Vocal cord cT1N0M0 Planned for Definite radiation therapy .He was treated with $6 \mathrm{Mv} \mathrm{X}$ Ray beam therapy to local site using 3D conformal technique to Total Dose of $63 \mathrm{~Gy}$ (225cGy in 28 fractions) for 5 days in week for 6 weeks. (Figure 2 Shows Dose distribution of PTV). 3D dose distribution and DVH of OAR and PTV are depicted in ()

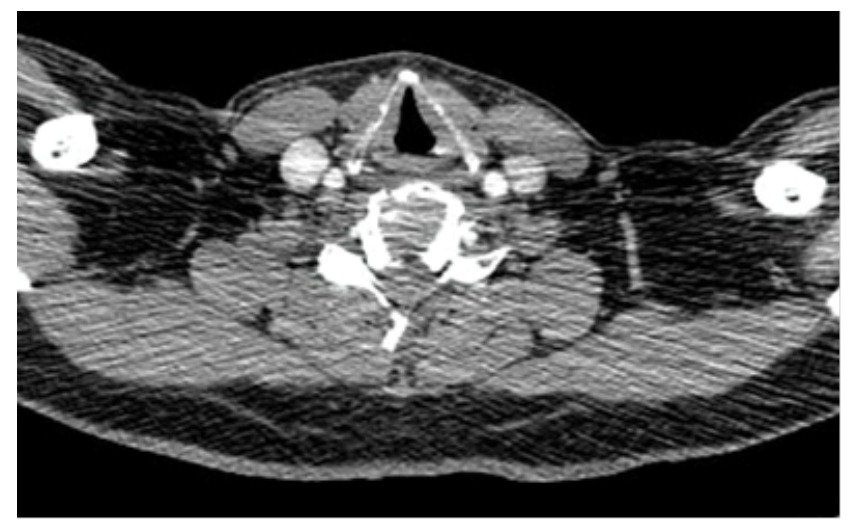

Figure 1.

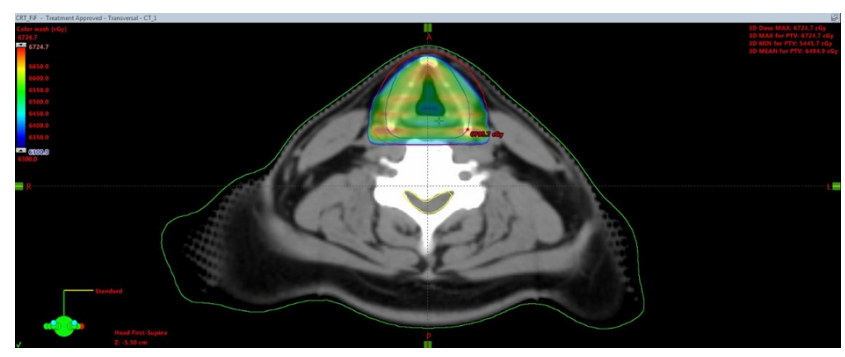

Figure 2.

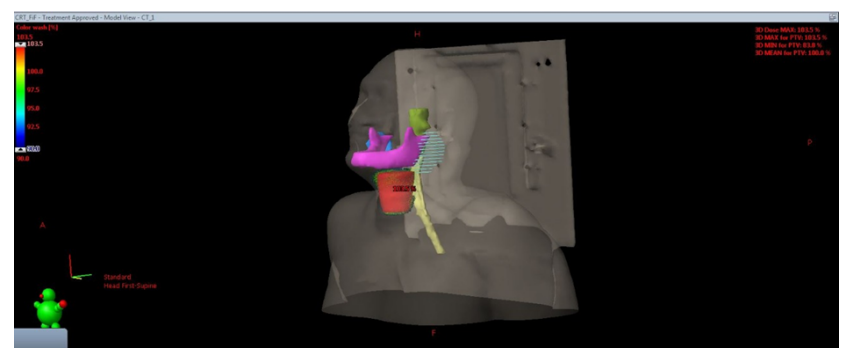

Figure 3.

\section{DISCUSSION:}

In Larynx, Squamous cell carcinoma was considered as most common type of malignant tumour. Carcinosarcoma consid-

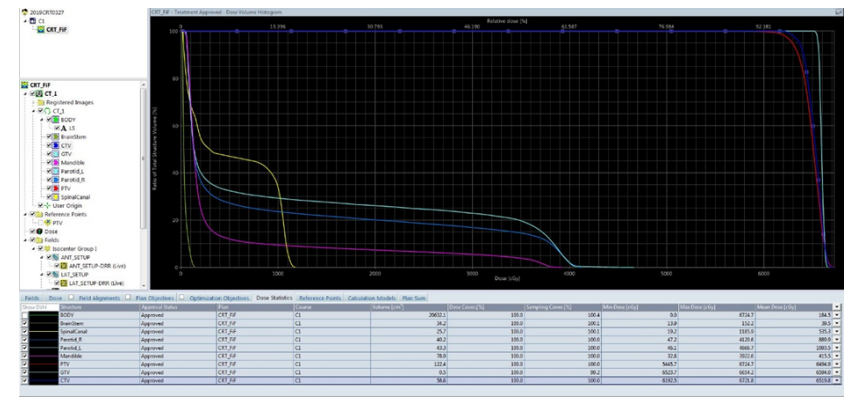

Figure 4.

ered to be monoclonal epithelial neoplasm with sarcomatous component derived from squamous epithelium with sarcomatous differentiation can arise in any site of body. It is a Biphasic tumour showing both carcinomatous (Epithelial component) and sarcomatous differentiation (Mesenchymal component) [2].

Most common site of origin in Head and Neck region is Oral Cavity (Salivary glands), Tonsil, Pharynx followed by Larynx. Etiology was not clearly known ,however strongly associated with chronic cigarette smoking and alcohol abuse. Most commonly noted in Males than Females in ratio of 12:1 with peak incidence in $6^{\text {th }}$ and $7^{\text {th }}$ decade [3]. Common symptoms being obstructive features Dysphagia, dysphonia, Odynophagia and Progressive Hoarseness of Voice [1].

Clinically tumor usually pedunculated polypoidal mass diagnosed histopathlogically [4] , usually managed surgically followed by radiation. In few cases literature review shows Endoscopic Co2 laser horizontal Partial laryngectomy was performed for early lesion -T1/T2. Chemotherapy is usually not preferred in sarcoma as they are chemoresistant.

In our case, initial clinical picture was not made out clearly, as he undergone biopsy elsewhere. Relook DLDP done in our institution showed small nodular growth of $3 \mathrm{~mm}$ which was removed in toto. Case discussed in Multidisciplinary board for final treatment plan as lesion in removed in toto. Surgery was deferred in view of T1 lesion .For Organ preservation preservation, considered for treatment with Definite Radiation Therapy. [5]

Although Prognosis of carcinosarcoma is controversial and reported to be worse than squamous cell carcinoma, early lesion has good 5-year survival from $65 \%$ to $95 \%$ [6]. Previous studies reported local recurrence ranges from $16 \%$ to $32 \%$ and cervical nodal metastasis ranges from $7 \%$ to $26 \%[7,8]$. Lambart et.al reported $5 \%$ distant metastasis to lung and soft tissue [9]. However, the patient should follow up with strict clinical and radiological follow up for recurrence of disease locally and distant.

\section{CONCLUSTON}

Carcinosarcoma is rare clinical scenario in Larynx, detected early due to Obstructive Symptoms. Usually managed Surgically with Laryngectomy. In our case patient was staged 
as cT1N0M0. In view of early leison, which was removed in toto with less likely of nodal metastasis, Our patient managed with Definite Radiation Therapy with Organ Preservation approach. Definite radiotherapy can be considered in patient with early lesion and whom surgery is deferred with good voice preservation. Need further studies and definite treatment protocol for management of carcinosarcoma of larynx.

\section{REFERENCES}

[1] Boamah H, Ballard B. A case report of spindle cell (Sarcomatoid) carcinoma of the larynx. Case. Rep Med;2012(2012):4.

[2] Katase N, Tamamura R, Gunduz M, Murakami J, Asaumi J, Tsukamoto G. A spindle cell carcinoma presenting with osseous metaplasia in the gingiva: A case report with immunohistochemical analysis. Head Face Med;2008(4).

[3] Facts NCIC. Head and Neck Cancer: Questions and Answers. Bethesda, MD: National Cancer Institute; 2012.

[4] Thompson LD, Wieneke JA, Miettinen M, Heffner DK. Spindle cell (Sarcomatoid) carcinomas of the larynx: A clinicopathologic study of 187 cases. Am J Surg Pathol;26:153-70.

[5] Lewis JE, Olsen KD, Sebo TJ. Spindle cell carcinoma of the larynx: Review of 26 cases including DNA content and immunohistochemistry. Hum Pathol; 1997.

[6] Hyams VJ. Spindle cell carcinoma of the larynx. Can J Otolaryngol;4:307-13.

[7] Lambert PR, Ward PH, Berci G. Pseudosarcoma of the larynx: A comprehensive analysis. Arch Otolaryngol; 1980.

[8] diagnosis of laryngeal spindle cell carcinoma and inflammatory myofibroblastic;.

[9] tumor-Report of two cases with similar morphology Diagn Pathology; 2007. 